\title{
A Case of Bordetella bronchiseptica Bacteremia in a Patient With Decompensated Liver Cirrhosis
}

\author{
Kok Hoe Chan ${ }^{1}$, Susanne O. Ajao ${ }^{1}$, Iyad Farouji ${ }^{1}$, Jihad Slim ${ }^{2}$ \\ 1. Internal Medicine, Saint Michael's Medical Center, Newark, USA 2. Infectious Diseases, Saint Michael's Medical \\ Center, Newark, USA
}

Corresponding author: Kok Hoe Chan, kokhoedelcos.chan@gmail.com

\begin{abstract}
Bordetella bronchiseptica is a rare cause of respiratory tract infection in humans, most commonly found in immunocompromised individuals exposed to infected animals. It colonizes the respiratory tract and can lead to infection in dogs, cats, rabbits, and others. In immunocompromised patients, it has been reported to result in life-threatening infections but rarely affects immunocompetent individuals. Here, we are the first to report a case B. bronchiseptica bacteremia in a patient with decompensated liver cirrhosis without known animal exposure.
\end{abstract}

Categories: Internal Medicine, Gastroenterology, Infectious Disease

Keywords: bordetella bronchiseptica, bacteremia, gram negative coccobacillus, decompensated liver cirrhosis

\section{Introduction}

Bordetella bronchiseptica, a gram-negative coccobacillus, is associated with infections in animals. Although it is a rare cause of human disease, cases including bacteremia and pneumonia have been reported in immunocompromised hosts [1-4]. Here, we present a rare case of a 63-year-old gentleman who was immunocompromised with liver cirrhosis and developed B. bronchiseptica bacteremia. To the best of our knowledge, no cases of $B$. bronchiseptica bacteremia in patients with liver cirrhosis have been reported in the literature.

Review began 03/12/2021 Review ended 03/15/2021 Published 03/17/2021

\section{() Copyright 2021}

Chan et al. This is an open access article distributed under the terms of the Creative Commons Attribution License CC-BY 4.0., which permits unrestricted use, distribution, and reproduction in any medium, provided the original author and source are credited.

\section{Case Presentation}

A 63-year-old African American male with past medical history of congestive heart failure, pulmonary sarcoidosis, non-alcoholic liver cirrhosis, chronic kidney disease, and hypertension presented with complains of shortness of breath and bilateral lower extremity swelling. He denied abdominal pain, upper respiratory tract symptoms, fever, chills, and rigor. He also denied recent exposure to pets and animals including dogs. Initial vital signs were stable. He was afebrile, normotensive, and saturated at 100\% on room air. Physical examination was significant for a markedly distended abdomen, which was soft and non-tender to light and deep palpation. Jugular venous distension was evident with extensive bilateral lower extremity edema with scrotal edema. There was no jaundice on examination. Chest X-ray was unremarkable with no infiltrates and consolidations. Initial laboratory investigation revealed a white count of 4.2 cells $/ \mathrm{mm}^{3}(4.4-$ 11.0 cells $\left./ \mathrm{mm}^{3}\right)$, hemoglobin of $10.7 \mathrm{~g} / \mathrm{dL}(13.5-17.5 \mathrm{~g} / \mathrm{dL})$, platelet of 188 cells $/ \mathrm{mm}^{3}\left(150-450\right.$ cells $\left./ \mathrm{mm}^{3}\right)$, lactic acid of $2.3 \mathrm{mmol} / \mathrm{L}(0.0-2.0 \mathrm{mmol} / \mathrm{L})$, creatinine of $1.7 \mathrm{mg} / \mathrm{dL}(0.6-1.2 \mathrm{mg} / \mathrm{dL})$, B-type natriuretic peptide of $823.71 \mathrm{pg} / \mathrm{mL}(0.0-100.0 \mathrm{pg} / \mathrm{mL})$, protein of $8.2 \mathrm{~g} / \mathrm{dL}(6.4-8.4 \mathrm{~g} / \mathrm{dL})$, and albumin of $2.6 \mathrm{~g} / \mathrm{L}$ (3.5-4.5 $\mathrm{g} / \mathrm{L})$. Liver function tests were mildly elevated with an aspartate aminotransferase of $43 \mathrm{U} / \mathrm{L}$ (10-36 U/L), alanine aminotransferase of $20 \mathrm{U} / \mathrm{L}$ (9-46 U/L), and alkaline phosphatase of $134 \mathrm{U} / \mathrm{L}$ (40-115 U/L). Total bilirubin was within normal limit. Coagulation profile showed slightly prolonged international normalized ratio of 1.46 .

He was admitted and managed for decompensated liver cirrhosis with diuresis to relieve the edema. He had undergone similar admissions in the past and also had multiple paracentesis done, the most recent was a month earlier, for symptomatic relief. A large-volume paracentesis was done during this hospitalization with the removal of $10 \mathrm{~L}$ of peritoneal fluid. Following paracentesis, he became hypotensive despite resuscitative efforts with appropriate albumin. Peritoneal fluid analysis revealed a white blood cell count of $72 / \mathrm{mm}^{3}, 49 \%$ lymphocyte differential, $44 \%$ neutrophil differential, protein of $2.6 \mathrm{~g} / \mathrm{dL}$, lactate dehydrogenase of $91 \mathrm{U} / \mathrm{L}$, and glucose of $113 \mathrm{mg} / \mathrm{dL}$. This analysis was consistent with a diagnosis of portal hypertension likely as a result of his liver cirrhosis, and spontaneous bacterial peritonitis was unlikely. No organism was isolated from peritoneal fluid cultures and gram stain showed no organism.

In the setting of his persistent hypotension, sepsis was considered, blood cultures were drawn, and meropenem was empirically started. Ultrasound of the abdomen was done to look for a source and showed moderate ascites with a contracted gallbladder with no evidence of cholecystitis (Figure 1). 


\section{Cureus}

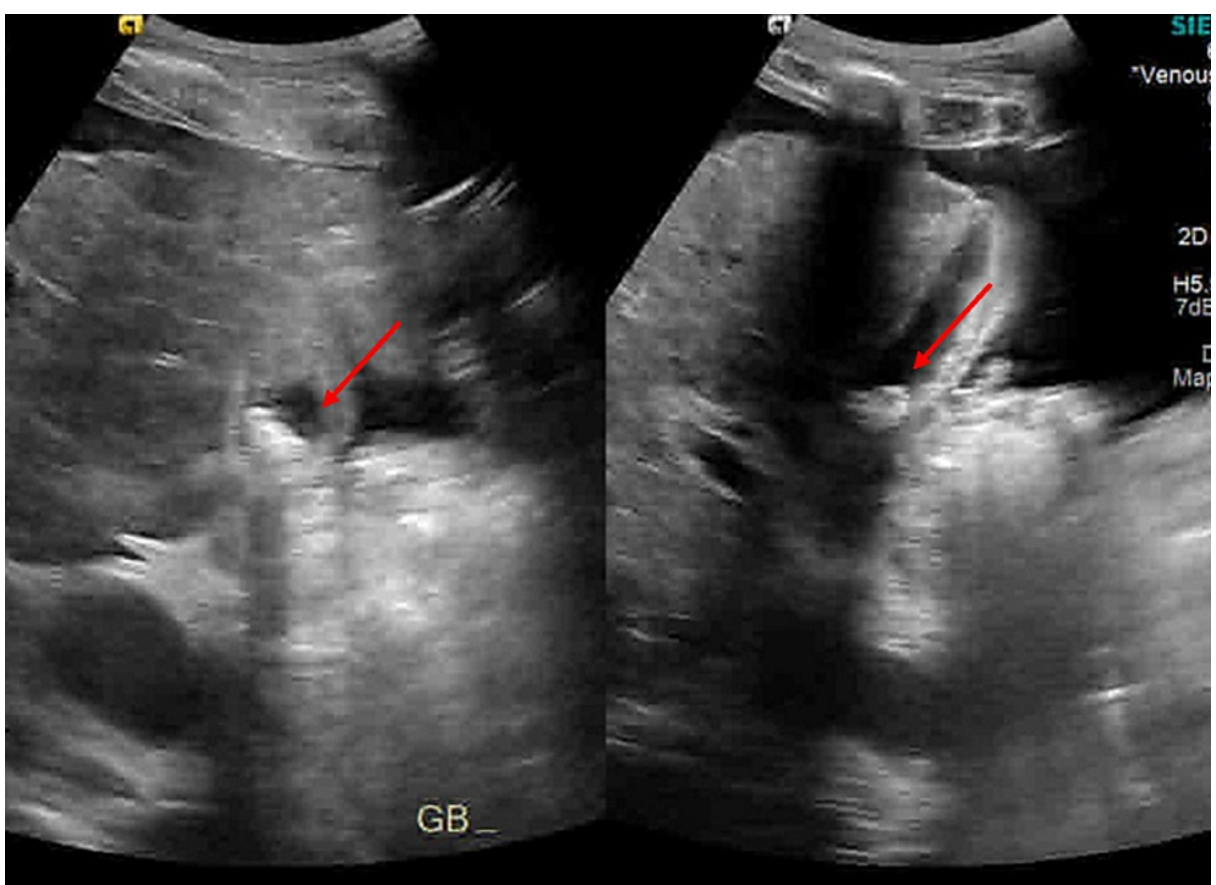

FIGURE 1: Right upper abdomen showed contracted gallbladder with gallstones, with no evidence of cholecystitis: transverse (left) and longitudinal (right) views.

Computed tomography of the abdomen was also done which showed cirrhosis and cholelithiasis with no evidence of intra-abdominal abscess (Figure 2). 


\section{Cureus}

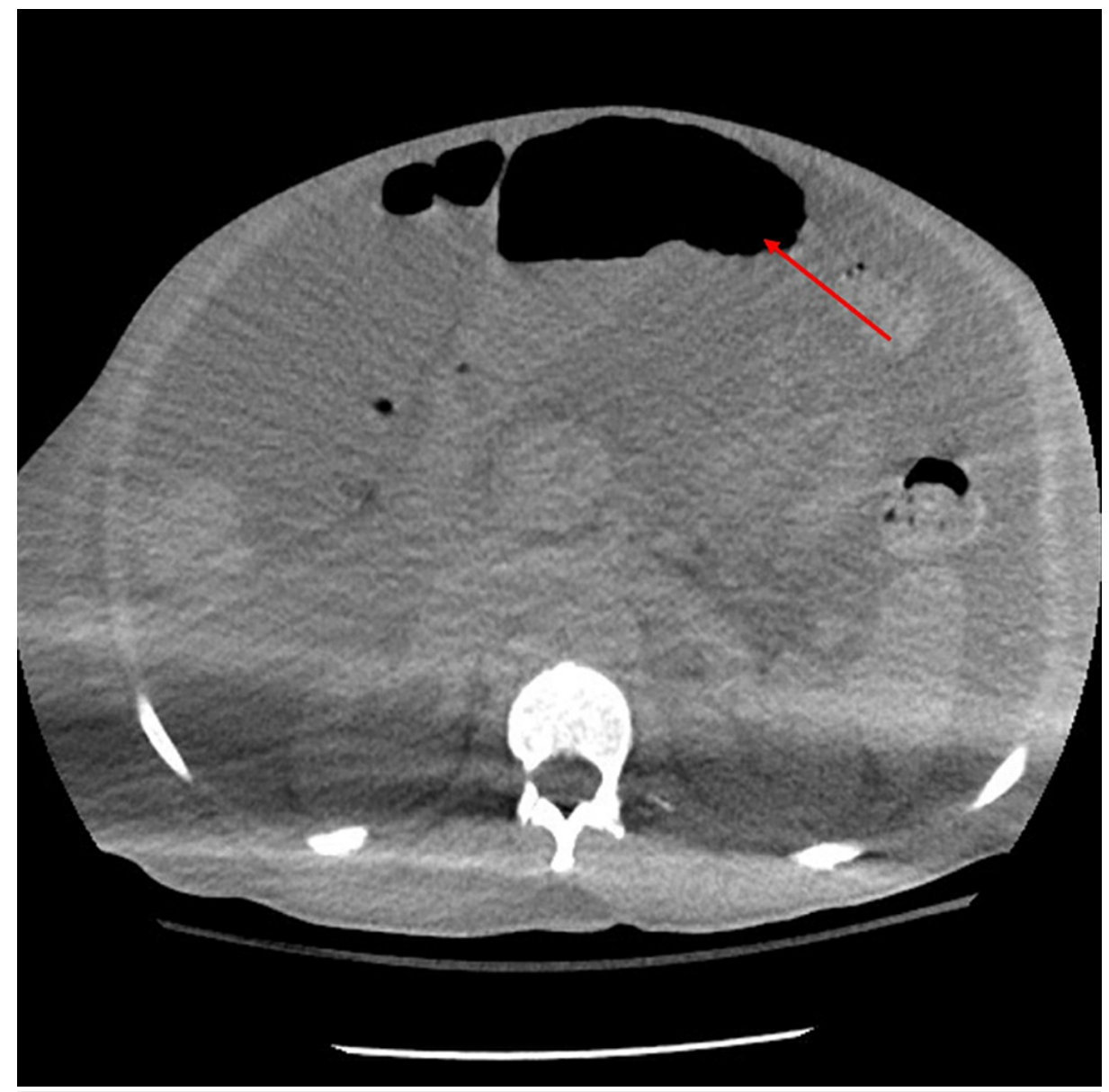

FIGURE 2: CT of the abdomen pelvis showed huge amount of ascites without evidence of intra-abdominal abscess.

$\mathrm{CT}$, computed tomography

A hepatobiliary iminodiacetic acid scan was done which revealed no biliary obstruction but delayed visualization of the gallbladder. Surgery was consulted and in the absence of clinical signs of cholecystitis with his multiple comorbidities, elective cholecystectomy was postponed, and ongoing antibiotic management was advised. B. bronchiseptica was isolated from blood cultures (Table 1). 


\section{Cureus}

$\begin{array}{ll}\text { Bordetella bronchiseptica } & \\ \text { Amikacin } & <=16 \text { Susceptible } \\ \text { Cefepime } & <=8 \text { Susceptible } \\ \text { Cefotaxime } & 8 \text { Susceptible } \\ \text { Ceftazidime } & 4 \text { Susceptible } \\ \text { Ceftriaxone } & <=8 \text { Susceptible } \\ \text { Ciprofloxacin } & <=1 \text { Susceptible } \\ \text { Gentamicin } & <=4 \text { Susceptible } \\ \text { Meropenem } & <=4 \text { Susceptible } \\ \text { Piperacillin + tazobactam } & <=16 \text { Susceptible } \\ \text { Tetracycline } & <=4 \text { Susceptible } \\ \text { Tobramycin } & <=4 \text { Susceptible } \\ \text { Trimethoprim + sulfamethoxazole } & <=2 / 38 \text { Susceptible }\end{array}$

TABLE 1: Culture and sensitivity of Bordetella bronchiseptica.

Echocardiogram was performed and did not show any vegetations (Figure 3).

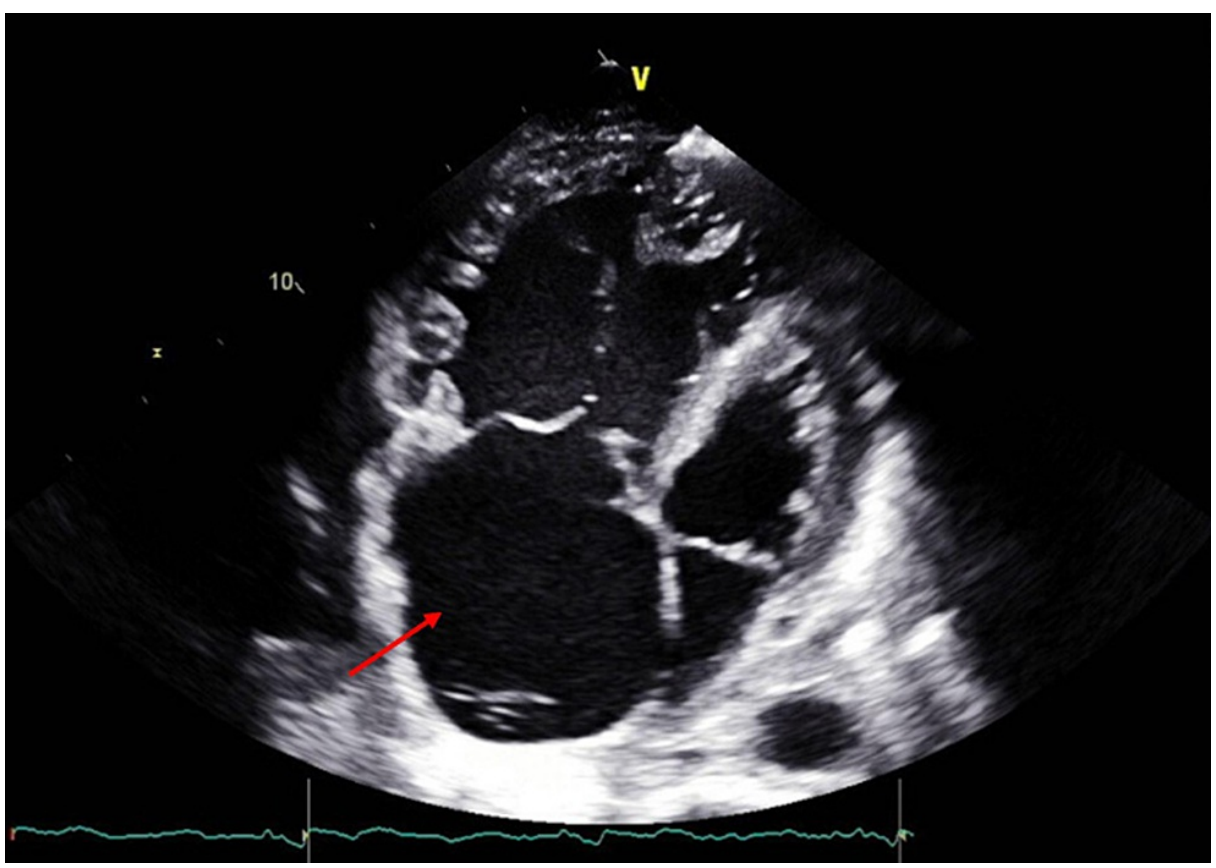

FIGURE 3: Echocardiogram four-chamber views showing severely dilated right atrium and right ventricle, with no evidence of vegetations.

Meropenem was stopped and based on a review of the available literature, we decided to start the patient on doxycycline for the bacteremia. Blood pressure stabilized after 48 hours and repeat blood cultures were negative. The patient tolerated doxycycline and was discharged with a seven-day course of oral doxycycline.

\section{Discussion}

Bordetella are gram-negative bacterial pathogens of mammals and birds. The most common species are $B$. pertusis, B. parapertusis, and B. bronchispetica [5]. B. pertussis, the causative agent of whooping cough, is a 
human-adapted variant of B. bronchiseptica. B. bronchispetica can colonize the upper respiratory tract of different animals [6]. It is very rare to isolate $B$. bronchispetica from human beings despite their frequent exposure to animals commonly colonized with this organism, including mice, dogs, cats, poultry, and livestock such as pigs. Most of the reported cases of $B$. bronchispetica were in severely immunocompromised patients [7]. It is believed that Bordetella species enter the respiratory tract through aerosol droplets [8]. In comparison to other pathogens, Bordetella have the ability to adhere efficiently to healthy, rapidly beating ciliated epithelial cells, where they begin to proliferate and produce factors that counteract host defenses, which prevents their elimination [9].

B. bronchiseptica is pathogenic in mammalian species and produces an endotoxin that is similar in chemical composition and physiological effects to those of other gram-negative microorganisms [10]. The bacterium can adhere to respiratory epithelial cells by using fimbriae and filamentous hemagglutinins. It invades respiratory epithelial cells and alveolar macrophages and diminishes the overall bactericidal ability of these cells by producing the enzyme adenylate cyclase. These virulence factors enable the organism to successfully colonize the respiratory tract $[11]$.

All B. bronchiseptica infections that have been reported in the literature were in immunocompromised patients who got the zoonotic infection through animal contact. $B$. bronchiseptica infections have been reported in patients with human immunodeficiency virus, hematological malignancy, or those on immunosuppressive medications [3,4]. Patients may present with a wide range of respiratory symptoms, ranging from asymptomatic to severe [3,4]. Patients infected with $B$. bronchiseptica typically present with classic symptoms of pneumonia, and in some cases, present with acute sinusitis and bronchitis. Our patient had extensive imaging done that did not show any abscesses in the body that may harbor this organism.

Patients with liver cirrhosis often have acquired immunodeficiency and systemic inflammation with increased risk or susceptibility to bacterial infections. This condition is recognized as cirrhosis-associated immune dysfunction [12]. Patients with liver cirrhosis not only have reduced number of neutrophils due to its sequestration by the enlarged spleen but also have been reported to have neutrophil dysfunction with defective phagocytosis of opsonized bacteria $[13,14]$. Nonetheless, to the best of our knowledge, there are no prior case report or studies on $B$. bronchiseptica in liver cirrhosis. We propose that the defective neutrophils may be the cause of bacteremia in our immunocompromised patient.

The diagnosis of $B$. bronchiseptica depends on positive cultures or polymerase chain reactions in patients with exposure to animals [15]. Blood cultures and bronchoalveolar lavage colony more than 104, in case of pneumonia, can help to determine whether $B$. bronchiseptica is a real infection or just airway colonization [16]. Regarding treatment, $B$. bronchiseptica can be treated as gram-negative non-fermentative pathogen [17]. Nonetheless, Schipper et al., despite the fact that $B$. bronchiseptica is an extracellular pathogen, the organism can invade and persist in eukaryotic cells, such as phagocytes and even epithelial cells; hence, it is very important to choose a medication with good intracellular penetration to prevent recurrent and chronic infections [18]. Various antibiotics can be used including aminoglycosides, quinolones, anti-pseudomonal penicillins, tetracycline, trimethoprim-sulfamethoxazole, imipenem, or a combination of erythromycin, ciprofloxacin and rifampin depending on the susceptibility $[19,20]$. Due to the rarity of this infection, there are no clear guidelines regarding the duration of the treatment. To our knowledge, we are presenting the first case in literature. Our patient had liver cirrhosis and presented with B. bronchiseptica bacteremia without any history of animal exposure and respiratory symptoms.

\section{Conclusions}

B. bronchiseptica is a gram-negative coccobacillus found commonly in the upper respiratory tract of animals. It can be transmitted to human beings causing zoonotic infection that may lead to life-threatening infection in those with underlying debilitation or impaired immunity (e.g., patients with neutropenia, diabetes, malnutrition, or transplant patients). We presented a very unique and rare case of liver cirrhosis patient who presented with $B$. bronchiseptica bacteremia without any respiratory symptoms and without direct exposure to animals. Due to the rarity of this case, there was no consensus guidelines for the treatment choice and duration. With the limited literature available, we treated the patient with doxycycline for seven days.

\section{Additional Information \\ Disclosures}

Human subjects: Consent was obtained or waived by all participants in this study. Conflicts of interest: In compliance with the ICMJE uniform disclosure form, all authors declare the following: Payment/services info: All authors have declared that no financial support was received from any organization for the submitted work. Financial relationships: All authors have declared that they have no financial relationships at present or within the previous three years with any organizations that might have an interest in the submitted work. Other relationships: All authors have declared that there are no other relationships or activities that could appear to have influenced the submitted work.

\section{References}


1. Powers HR, Shah K: Bordetella bonchiseptica blood stream infection in a renal transplant patient . Transpl Infect Dis. 2017, 19:12774. 10.1111/tid.12774

2. Mazumder SA, Cleveland KO: Bordetella bronchiseptica bacteremia in a patient with AIDS. South Med J. 2010, 103:934-5. 10.1097/SMJ.0b013e3181ebcdbc

3. Buggy BP, Brosius FC, Bogin RM, Koller CA, Schaberg DR: Bordetella bronchiseptica pneumonia in a patient with chronic lymphocytic leukemia. South Med J. 1987, 80:1187-9. 10.1097/00007611-198708090-00028

4. Huebner ES, Christman B, Dummer S, Tang YW, Goodman S: Hospital-acquired Bordetella bronchiseptica infection following hematopoietic stem cell transplantation. J Clin Microbiol. 2006, 44:2581-3. 10.1128/JCM.00510-06

5. Goodnow RA: Biology of Bordetella bronchiseptica. Microbiol Rev. 1980, 44:722-38. 10.1128/mmbr.44.4.722-738.1980

6. Nagano H, Nakai T, Horiguchi Y, Kume K: Isolation and characterization of mutant strains of Bordetella bronchiseptica lacking dermonecrotic toxin-producing ability. J Clin Microbiol. 1988, 26:1983-7. 10.1128/jcm.26.10.1983-1987.1988

7. Woolfrey BF, Moody JA: Human infections associated with Bordetella bronchiseptica. Clin Microbiol Rev. 1991, 4:243-55. 10.1128/CMR.4.3.243

8. Trainor EA, Nicholson TL, Merkel TJ: Bordetella pertussis transmission. Pathog Dis. 2015, 73:68. 10.1093/femspd/ftv068

9. Forde CB, Shi X, Li J, Roberts M: Bordetella bronchiseptica-mediated cytotoxicity to macrophages is dependent on bvg-regulated factors, including pertactin. Infect Immun. 1999, 67:5972-8. 10.1128/iai.67.11.5972-5978.1999

10. Bemis DA, Wilson SA: Influence of potential virulence determinants on Bordetella bronchiseptica-induced ciliostasis. Infect Immun. 1985, 50:35-42. 10.1128/iai.50.1.35-42.1985

11. Guetter SD, Eiteman MA: Production of biomass and filamentous hemagglutinin by Bordetella bronchiseptica. Bioprocess Biosyst Eng. 2014, 37:115-23. 10.1007/s00449-013-0977-4

12. Albillos A, Lario M, Álvarez-Mon M: Cirrhosis-associated immune dysfunction: distinctive features and clinical relevance. J Hepatol. 2014, 61:1385-96. 10.1016/j.jhep.2014.08.010

13. Fiuza C, Salcedo M, Clemente G, Tellado JM: In vivo neutrophil dysfunction in cirrhotic patients with advanced liver disease. J Infect Dis. 2000, 182:526-33. 10.1086/315742

14. Tritto G, Bechlis Z, Stadlbauer V, et al.: Evidence of neutrophil functional defect despite inflammation in stable cirrhosis. J Hepatol. 2011, 55:574-81. 10.1016/j.jhep.2010.11.034

15. Hozbor D, Fouque F, Guiso N: Detection of Bordetella bronchiseptica by the polymerase chain reaction. Res Microbiol. 1999, 150:333-41. 10.1016/s0923-2508(99)80059-x

16. Gisel JJ, Brumble LM, Johnson MM: Bordetella bronchiseptica pneumonia in a kidney-pancreas transplant patient after exposure to recently vaccinated dogs. Transpl Infect Dis. 2009, 12:73-6. 10.1111/j.13993062.2009.00451.x

17. Gomez L, Grazziutti M, Sumoza D, Beran M, Rolston K: Bacterial pneumonia due to Bordetella bronchiseptica in a patient with acute leukemia. Clin Infect Dis. 1998, 26:1002-3. 10.1086/517630

18. Schipper H, Krohne GF, Gross R: Epithelial cell invasion and survival of Bordetella bronchiseptica . Infect Immun. 1994, 62:3008-11. 10.1128/IAI.62.7.3008-3011.1994

19. Matic NA, Bunce PE: Isolation of Bordetella bronchiseptica from blood and a pancreatic abscess . J Clin Microbiol. 2015, 53:1778-80. 10.1128/JCM.00175-15

20. Katzenstein DA, Ciofalo L, Jordan MC: Bordetella bronchiseptica bacteremia. West J Med. 1984, 140:96-8. 